\title{
Targeting fatty acid $\beta$-oxidation impairs monocyte differentiation and prolongs heart allograft survival
}

\author{
Yuehui Zhu, ${ }^{1}$ Hao Dun, ${ }^{1}$ LiYe, ${ }^{1}$ Yuriko Terada, ${ }^{1}$ Leah P. Shriver, ${ }^{2}$ Gary J. Patti, ${ }^{2,3}$ Daniel Kreisel, ${ }^{1,4}$ \\ Andrew E. Gelman, ${ }^{1,4}$ and Brian W. Wong ${ }^{1}$ \\ ${ }^{1}$ Department of Surgery, ${ }^{2}$ Department of Chemistry, ${ }^{3}$ Department of Medicine, and ${ }^{4}$ Department of Pathology \& \\ Immunology, Washington University School of Medicine, St. Louis, Missouri, USA.
}

\begin{abstract}
Monocytes play an important role in the regulation of alloimmune responses after heart transplantation (HTx). Recent studies have highlighted the importance of immunometabolism in the differentiation and function of myeloid cells. While the importance of glucose metabolism in monocyte differentiation and function has been reported, a role for fatty acid $\beta$-oxidation (FAO) has not been explored. Heterotopic HTx was performed using hearts from BALB/c donor mice implanted into C57BL/ 6 recipient mice and treated with etomoxir (eto), an irreversible inhibitor of carnitine palmitoyltransferase 1 (Cpt1), a rate-limiting step of FAO, or vehicle control. FAO inhibition prolonged HTx survival, reduced early $\mathrm{T}$ cell infiltration/activation, and reduced DC and macrophage infiltration to heart allografts of eto-treated recipients. ELISPOT demonstrated that splenocytes from eto-treated $\mathrm{HTx}$ recipients were less reactive to activated donor antigen-presenting cells. FAO inhibition reduced monocyte-to-DC and monocyte-to-macrophage differentiation in vitro and in vivo. FAO inhibition did not alter the survival of heart allografts when transplanted into Ccr2deficient recipients, suggesting that the effects of FAO inhibition were dependent on monocyte mobilization. Finally, we confirmed the importance of FAO on monocyte differentiation in vivo using conditional deletion of Cpt1a. Our findings demonstrate that targeting FAO attenuates alloimmunity after $\mathrm{HTx}$, in part through impairing monocyte differentiation.
\end{abstract}

Authorship note: $\mathrm{YZ}$ and $\mathrm{HD}$ contributed equally to this work and are co-first authors.

Conflict of interest: The authors have declared that no conflict of interest exists.

Copyright: () 2022, Zhu et al. This is an open access article published under the terms of the Creative Commons Attribution 4.0 International License.

Submitted: May 21, 2021

Accepted: March 2, 2022

Published: March 3, 2022

Reference information: /CI Insight. 2022;7(7):e151596.

https://doi.org/10.1172/jci.

insight.151596.

\section{Introduction}

Heart transplantation (HTx) is the preferred treatment for numerous patients with end-stage heart failure; it provides a median survival of approximately 12 years and a significant improvement in quality of life $(1,2)$. Calcineurin inhibitors (FK506; cyclosporine) remain the primary immunosuppressive agent, despite the advent of newer immunosuppressants, such as mTOR inhibitors (rapamycin [sirolimus]; everolimus) or costimulation blockers (anti-CTLA4-immunoglobulin; belatacept). All these immunosuppressive agents (including the adjuvant therapies of corticosteroids and mycophenolic acid) mechanistically target $\mathrm{T}$ cell proliferation, and, despite robust suppression of acute $\mathrm{T}$ cell responses that have led to dramatic reductions in acute rejection, chronic allograft rejection remains.

During acute inflammation, classical monocytes are mobilized from the bone marrow and migrate to sites of injury (3). Monocytes can respond to factors such as granulocyte macrophage colony-stimulating factor (GM-CSF) or macrophage colony-stimulating factor (M-CSF), among other factors, to differentiate into monocyte-derived cells that have phenotypic similarities to DCs and macrophages (4-7). In acute kidney allograft rejection, monocyte infiltration has been quantitatively associated with renal dysfunction (8), and monocyte-mediated acute renal rejection has been reported after inhibition of $\mathrm{T}$ cell infiltration by alemtuzumab (9). A higher number of $\mathrm{CD}_{1} 6^{+}$monocytes before transplant was associated with higher risk of acute rejection after kidney transplantation (10). The monocyte-macrophage lineage is central to allograft rejection, contributing to alloimmunity via multiple mechanisms, including antigen processing/presentation, costimulation, and proinflammatory cytokine production $(11,12)$. CD16 ${ }^{+}$monocyte infiltration and polarized M2 macrophages in endomyocardial biopsies have been associated with acute cellular rejection in human heart transplants (13). Monocyte-derived DCs have also been reported to impair early graft function in islet transplantation (14) and can promote cardiac allograft rejection (15). 
Immunometabolism, the study of the role of metabolic pathways in immune cells, has recently emerged as a central regulator of immune cell differentiation and function (16-22). To date, a role for fatty acid $\beta$-oxidation (FAO) has not been explored in monocyte differentiation. Much of our insight on the role of oxidative metabolism has been garnered through in vitro analysis of cells using extracellular flux analysis, which only provides an assessment of oxygen consumption and extracellular (media) acidification as a surrogate for lactate production based on substrate utilization in the presence and absence of metabolic inhibitors $(16,20,23)$. In this study, we aimed to test the effect of pharmacological inhibition of FAO using etomoxir (eto), an irreversible inhibitor of carnitine palmitoyltransferase 1 (Cpt1), a rate-limiting step of FAO, in a murine model of acute heart allograft rejection. Further, we verified the importance of FAO in monocyte differentiation in vivo using conditional deletion of Cpt1a.

\section{Results}

Pharmacological inhibition of FAO extends HTx survival. To investigate the role of FAO in HTx rejection, we performed syngeneic $(\mathrm{C} 57 \mathrm{BL} / 6[\mathrm{~B} 6] \rightarrow \mathrm{B} 6)$ and allogeneic $(\mathrm{BALB} / \mathrm{c}[\mathrm{BALB}] \rightarrow \mathrm{B} 6)$ murine heterotopic cardiac transplants. Pharmacological inhibition of FAO with eto starting on the day of transplantation significantly prolonged allograft survival compared with treatment with vehicle control (ctrl) (median graft survival: 13 versus 8 days in eto- vs. ctrl-treated HTx; Figure 1A). Histological analysis 4 days after transplant revealed reduced immune cell infiltration in eto-treated HTx recipients (Figure 1B). To further define the cellular infiltrate preceding acute heart allograft rejection, flow cytometric analysis was performed on day 4 after transplant. This revealed a significant reduction in the abundance of $\mathrm{T}$ cells, activated $\mathrm{T}$ cells, $\mathrm{CD} 4^{+} \mathrm{T}$ cells, $\mathrm{CD} 8^{+} \mathrm{T}$ cells, and Foxp $3^{+} \mathrm{CD} 4^{+} \mathrm{T}$ cells consistent with the phenotype of regulatory $\mathrm{CD}^{+} \mathrm{T}$ cells (Tregs) in transplanted hearts from eto-treated HTx recipients compared with ctrl-treated HTx recipients (Figure 1, C-G). We did not observe a difference in the ratio of $\mathrm{CD}^{+}$to $\mathrm{CD} 8^{+}$ $\mathrm{T}$ cells in transplanted hearts between the 2 groups (Supplemental Figure 1A; supplemental material available online with this article; https://doi.org/10.1172/jci.insight.151596DS1). We also observed a significant reduction in $\mathrm{CD} 11 \mathrm{~b}^{+} \mathrm{CD} 64^{+} \mathrm{CD} 24^{-}$macrophages and $\mathrm{CD} 11 \mathrm{c}^{+} \mathrm{MHCII}^{+} \mathrm{CD} 64^{-} \mathrm{DCs}$ in transplanted hearts from eto-treated HTx recipients compared with ctrl-treated HTx recipients (Figure 2, A and B). In line with this observation, allografts from eto-treated HTx recipients had significant reductions in both recipient-derived macrophages and DCs (Figure 2, C and D). Analysis of FAO inhibition in syngrafts did not reveal any significant differences in infiltrating leukocytes relative to the syngrafts receiving vehicle ctrl (Supplemental Figure 1, B-H).

Inhibition of FAO reduces alloreactivity after HTx. We observed significant reduction in IFN- $\gamma, \mathrm{TNF}-\alpha$, and IL-2 production in splenocytes isolated from eto-treated HTx compared with ctrl-treated HTx recipients when stimulated with donor- but not recipient-derived antigen-presenting cells (APCs) (Figure 3 and Supplemental Figure 2, A-C). In vitro inhibition of FAO in splenocytes from ctrl-treated HTx recipients did not alter IFN- $\gamma$ production when stimulated with positive ctrl (concanavalin A) or donor-derived APCs (Supplemental Figure 2, D and E), indicating that the effect of FAO in vivo is not likely due to direct $\mathrm{T}$ cell inhibition. Furthermore, FAO inhibition in splenocytes that were isolated from ctrl-treated HTx allograft recipients did not alter their proliferative responses after stimulation with donor-derived APCs (Supplemental Figure 2F).

FAO inhibition impairs monocyte differentiation after HTx. We next sought to investigate whether FAO plays a role in monocyte differentiation. To this end, we differentiated bone marrow-derived monocytes toward DC (GM-CSF + IL4) and macrophage (M-CSF) fates in the presence or absence of $100 \mu \mathrm{M}$ eto for 7 days. Pharmacological inhibition of FAO significantly impaired the generation of cells with dendritic (Figure 4A) and macrophage (Figure 4B) phenotypes. Analysis of proliferation did not reveal any significant differences between eto- and ctrl-treated cells toward either fate (Supplemental Figure 3, A and B). Our investigation of central carbon flux in monocytes showed a significant increase in FAO flux after 48-hour incubation following either GM-CSF + IL4 or M-CSF stimulation, which was sharply inhibited by pharmacological inhibition of FAO (Figure 4C). Inhibition of FAO flux in monocytes by eto was confirmed for a range of doses from 5 to $100 \mu \mathrm{M}$ (Supplemental Figure 3C). Furthermore, Cpt1 activity assay was used to verify the ability of eto to impair both palmitate and palmitoyl-CoA transport into isolated mitochondria from murine monocytes (Figure 4, D and E). Stimulation of monocytes with either GM-CSF + IL4 or M-CSF significantly increased both glycolytic flux (Supplemental Figure 3D) and cell proliferation (Supplemental Figure 3E). We did not observe any increased cell death due to inhibition of FAO, as assessed by 
A
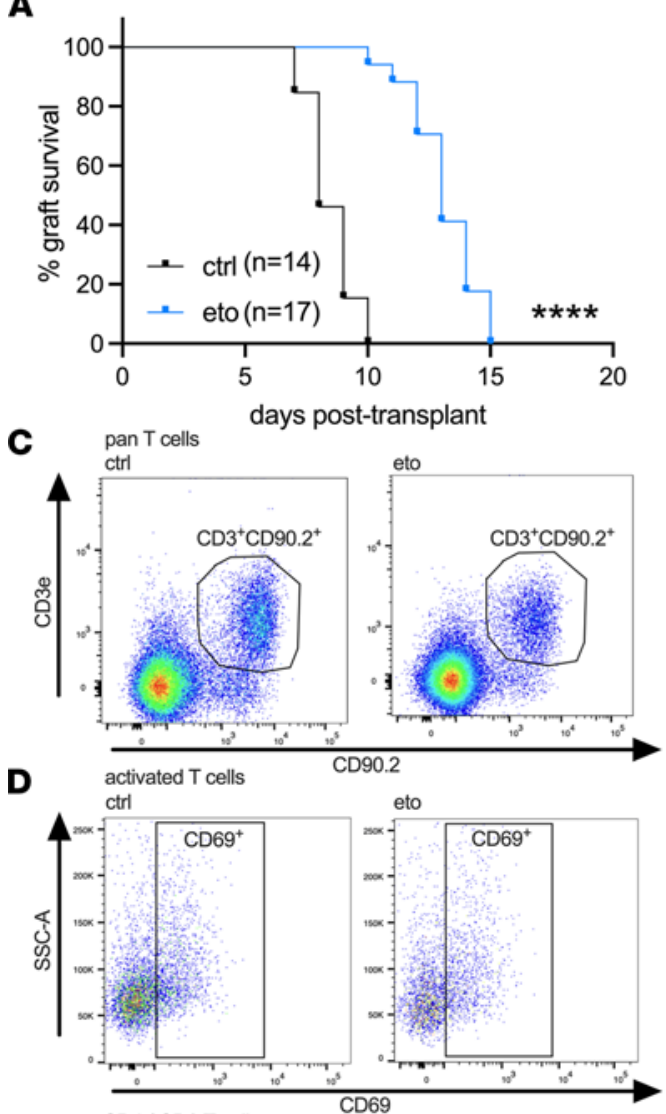
CD4 / CD8 T cells

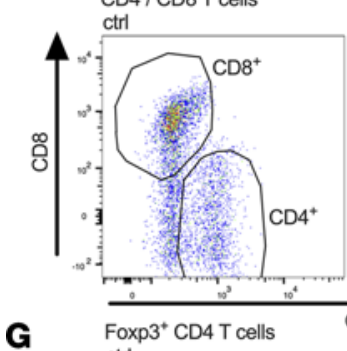

G

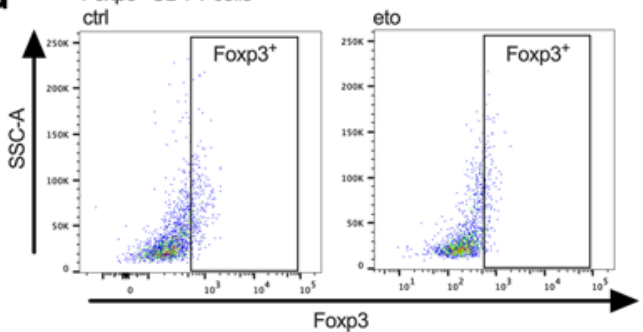

B

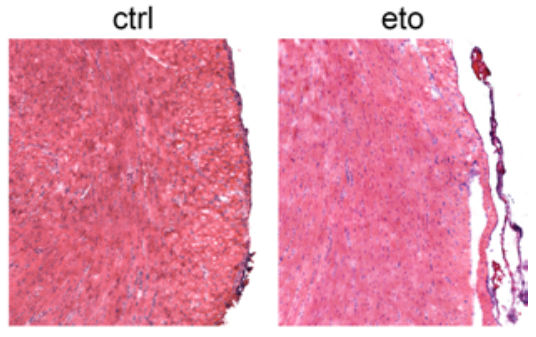

Balb/c to C57BI/6 HTx (4d)
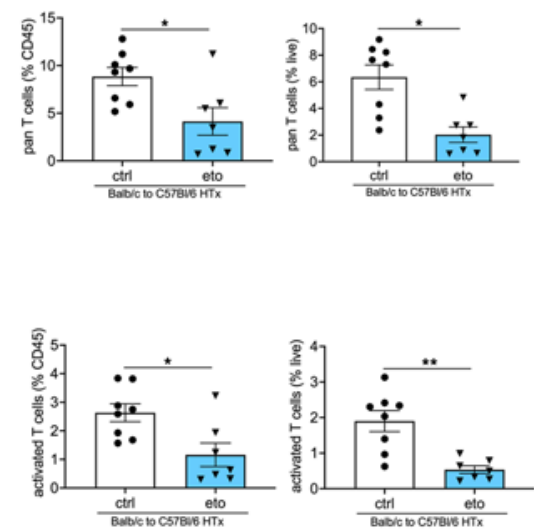

E
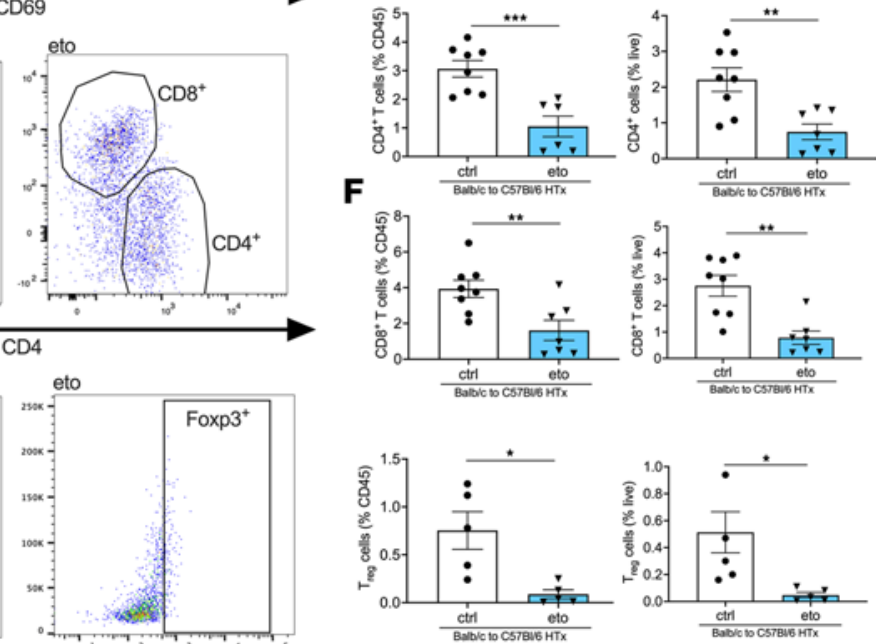

Figure 1. FAO inhibition improves HTx survival and decreases T cells in the transplanted heart. (A) Comparison of graft survival in vehicle-treated (ctrl-treated) or etomoxir-treated (eto-treated) BALB $\rightarrow B 6$ heart allografts. The survival benefit was significant $\left({ }^{* * *} P<0.0001\right.$ by Mantel-Cox test). (B) Representative micrographs from 4 days after transplant showing reduced immune cell infiltration in allografts from eto- compared with ctrl-treated HTx (original magnification, $\times 20$ ). (C-C) Flow cytometric assessment in transplanted hearts from BALB $\rightarrow$ B6 heart allografts 4 days after transplant, shown as a percentage of CD45+ cells (left graph) or live cells (right graph). (C) Pan T cells assessed by CD3+CD90.2+ cells (ctrl, $n=8$; eto, $n=7$ ). (D) Activated T cells assessed by CD69 ${ }^{+}$CD3 $3^{+}$CD90.2 $2^{+}$cells (ctrl, $n$

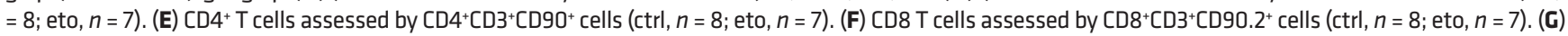
Tregs assessed by Foxp3 ${ }^{+} \mathrm{CD} 4{ }^{+} \mathrm{CD} 3^{+} \mathrm{CD} 90.2^{+}$cells (ctrl, $n=5$; eto, $n=5$ ). Data are shown as mean \pm SEM. ${ }^{*} P<0.05 ;{ }^{* *} P<0.01$; ${ }^{* *} P<0.001$ by $t$ test.

lactate dehydrogenase (LDH) enzyme release, and FAO inhibition in GM-CSF + IL4-treated monocytes reduced LDH release, suggesting decreased cell death (Figure $4 \mathrm{~F}$ ).

To investigate the role of FAO on monocyte differentiation in vivo, we adoptively transferred $\mathrm{CD} 45.1^{+}$ monocytes at the time of HTx into eto- or ctrl-treated recipients (Figure 5A). We observed significant 
A
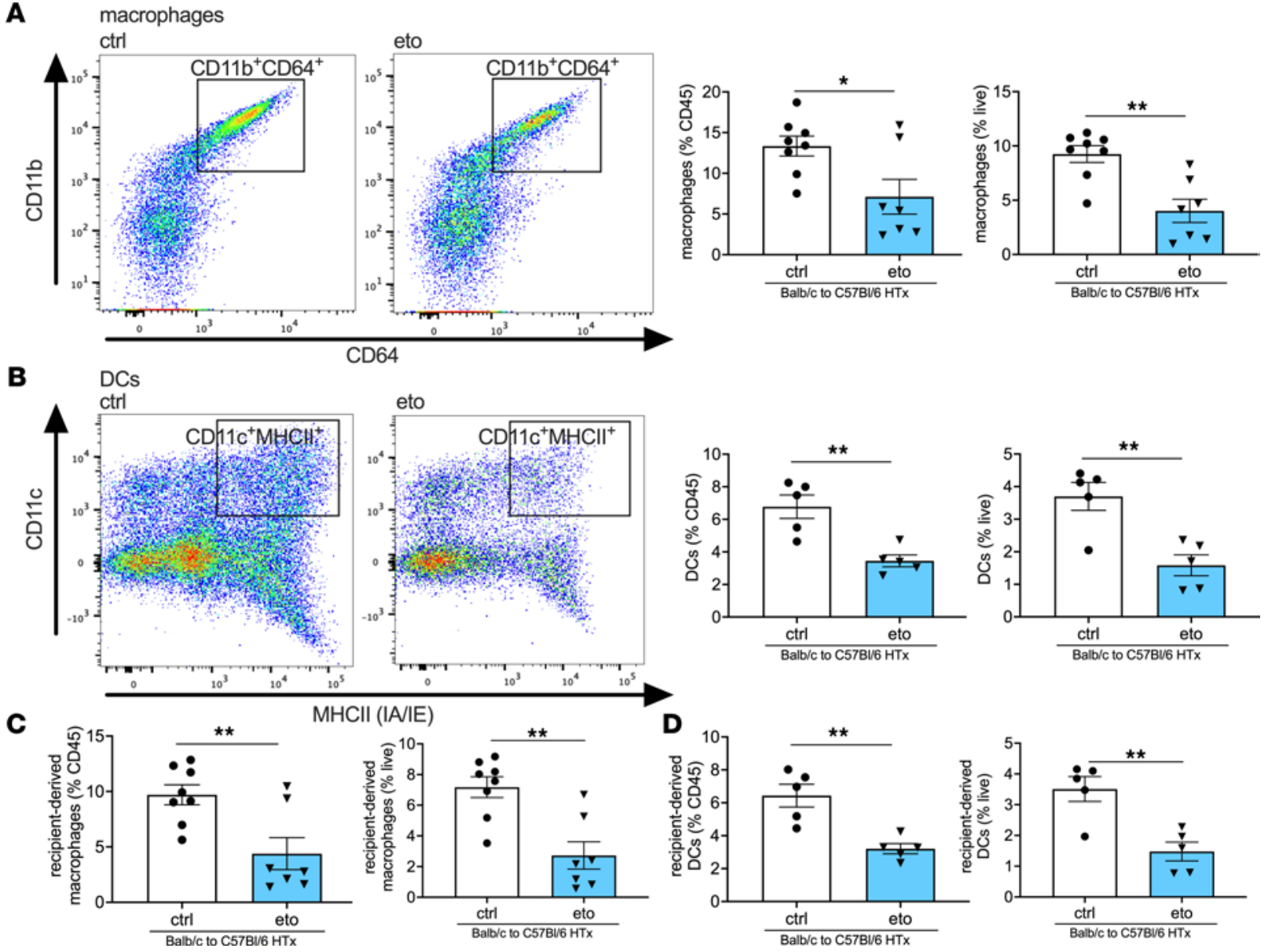

Figure 2. FAO inhibition reduces macrophages and DCs in the transplanted heart. Flow cytometric assessment in vehicle-treated (ctrl-treated) or etomoxir-treated (eto-treated) BALB $\rightarrow$ B6 heart allografts 4 days after transplant shown as a percentage of CD45+ cells (left graph) or live cells (right graph). (A) Macrophages assessed by CD116+CD64+CD24- cells (ctrl, $n=8$; eto, $n=7$ ). (B) DCs assessed by CD11c ${ }^{+}$MHCII ${ }^{+}$cells (ctrl, $n=5$; eto, $n=5$ ). (C) Recipient-derived macrophages assessed by IA-b+CD11 ${ }^{+}$CD64 ${ }^{+}$CD24- cells (ctrl, $n=8$; eto, $n=7$ ). (D) Recipient-derived DCs assessed by IA-b+CD11c ${ }^{+} \mathrm{MHCII}^{+}$cells (ctrl, $n=5$; eto, $n=5$ ). Data are shown as mean $\pm \mathrm{SEM}$. ${ }^{*} P<0.05$; ${ }^{* *} P<0.01$ by $t$ test.

reductions in $\mathrm{CD} 45.1^{+} \mathrm{DCs}$ and $\mathrm{CD} 45.1^{+}$macrophages in the allograft hearts of eto-treated recipients compared with that in ctrl-treated recipients (Figure 5, B and C). There were comparable abundances of Ki-67 proliferating monocyte-derived cells in allografts between eto- and ctrl-treated recipients (Supplemental Figure 4, A and B), and no significant differences in the relative abundance of $\mathrm{CD} 45.1^{+}$cells in transplanted hearts, native hearts, spleens, or mediastinal lymph nodes (Supplemental Figure 4C). Moreover, the relative abundances of $\mathrm{CD} 45.1^{+} \mathrm{DCs}$ or $\mathrm{CD} 45.1^{+}$macrophages were comparable in the native hearts, spleens, and mediastinal lymph nodes of eto- and ctrl-treated HTx recipients (Supplemental Figure 4, D and E). Analysis of CD $45.2^{+}$DCs and macrophages revealed a significant reduction in these populations in transplanted hearts from eto-treated HTx recipients compared with those from ctrl-treated HTx recipients (Supplemental Figure 4, F and G).

$H T x$ survival benefit from $\mathrm{FAO}$ inhibition is abrogated in $\mathrm{Crr}^{-1-}$ recipients. To further assess whether the HTx survival benefit observed from pharmacological inhibition of FAO was related to its effects on monocytes, we transplanted BALB hearts into B6 Ccr2-deficient $\left(\mathrm{Crr}^{---}\right)$recipients. We and others have shown that monocyte mobilization from the bone marrow is dependent on Ccr2 (24-26). Consistent with observations from previous reports, we observed a modest prolongation in allograft survival when recipients lack Ccr2 $(27,28)$. Importantly, heart allograft survival in $\mathrm{Ccr}^{---}$recipients was independent of FAO inhibition (median HTx survival, 14 versus 14 days after transplant in ctrl- versus eto-treated $\mathrm{Ccr}^{2---} \mathrm{HTx}$; Figure 6A). Of note, the median survival of hearts that were transplanted into either ctrl- and eto-treated $\mathrm{C}_{\mathrm{cr}} 2^{--1} \mathrm{HTx}$ recipients was comparable to BALB hearts transplanted into WT B6 allogeneic hosts that were treated with eto. We observed similar relative abundances of total and activated $\mathrm{T}$ cells within transplanted hearts 
A

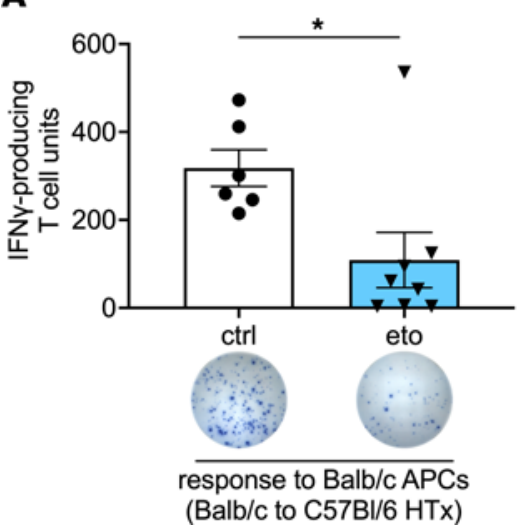

B

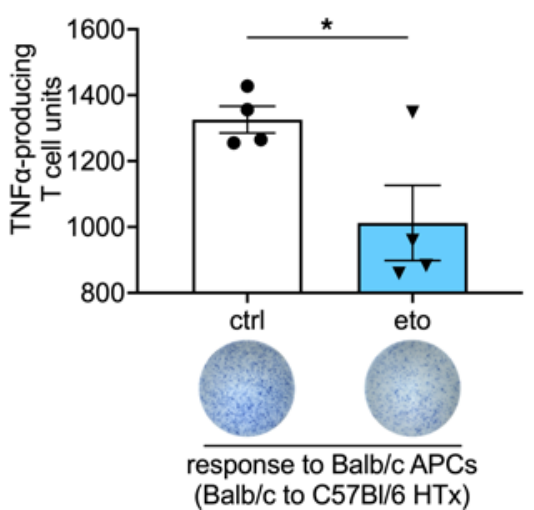

C

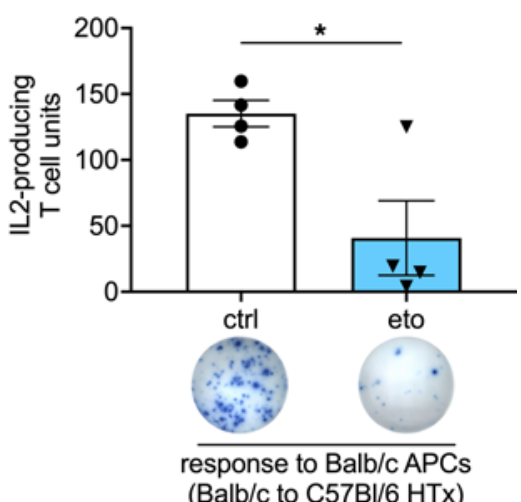

Figure 3. FAO inhibition reduces alloreactivity after HTX. ELISPOT assay performed using splenocytes procured 4 days after transplantation from vehicle-treated (ctrl-treated) or etomoxir-treated (eto-treated) BALB $\rightarrow$ B6 heart allografts. Samples were stimulated with donor-derived (BALB) APCs and assessed for the production of (A) IFN- $\gamma$ (ctrl, $n=6$; eto, $n=8$ ), (B) TNF- $\alpha$ (ctrl, $n=4$; eto, $n=4$ ), or (C) IL-2 (ctrl, $n=4$; eto, $n=4$ ). Data are shown as mean \pm SEM. ${ }^{*} P<0.05$ by $t$ test.

between ctrl- and eto-treated $\mathrm{Ccr}^{-1-} \mathrm{HTx}$ recipients, which were also comparable to our observations in cardiac allografts after transplantation into eto-treated B6 recipients (Figure 6, B and C). Additionally, there were no significant differences in the relative abundances of DCs, macrophages, $\mathrm{CD} 4^{+}$or $\mathrm{CD} 8^{+} \mathrm{T}$ cells, or Tregs in allografts in eto- versus ctrl-treated $\mathrm{Ccr}^{---} \mathrm{HTx}$ recipients, suggesting that the reduction in these immune cell populations induced by FAO inhibition in BALB to B6 HTx were secondary to the presence of $\mathrm{Ccr}^{+}$cells in the transplant recipient (Figure 6, D and E, and Supplemental Figure 5, A-C). As expected, heart allografts from $\mathrm{Ccr}^{-/-} \mathrm{HTx}$ recipients had significantly reduced numbers of DCs compared with heart allografts from BALB $\rightarrow$ B6 HTx recipients, with a trend toward reduced numbers of macrophages (Figure $6, \mathrm{D}$ and E). Further, investigation of recipient-derived populations of DCs and macrophages revealed a robust decrease in these populations in BALB $\rightarrow \mathrm{Ccr}^{---}$HTx recipients (Supplemental Figure 5, D and E). Finally, assessment of $\mathrm{T}$ cell alloreactivity showed no significant differences between splenocytes from ctrl- and eto-treated $\mathrm{Ccr}^{2--} \mathrm{HTx}$ recipients when stimulated with donor- or recipient-derived APCs (Figure 6F and Supplemental Figure 5F).

Genetic deletion of Cpt1a in monocytes impairs monocyte differentiation after HTx. To further confirm the importance of FAO in monocyte differentiation, we generated transgenic mice with conditional deletion of

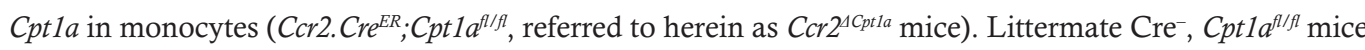
were used as ctrls (referred to herein as WT mice). We adoptively transferred WT or $C$ cr2 $2^{2 \mathrm{Cptta}}$ monocytes into HTx recipients (CD45.1 $1^{+}$BALB heart to $\mathrm{CD} 45.1^{+} \mathrm{B} 6$ recipient) at the time of transplantation (Figure $7 \mathrm{~A}$ ) and observed significant reductions in the relative abundance of $\mathrm{CD} 45.2^{+} \mathrm{DCs}$ and $\mathrm{CD} 45.2^{+}$macrophages in transplanted hearts from recipients adoptively transferred with $\mathrm{C} c 2^{4{ }^{4} p t t a}$ compared with WT monocytes (Figure 7, B and C). In vivo deletion of Cpt1a in Ccr2-expressing cells did not alter monocyte numbers in the bone marrow (Supplemental Figure 6A). Assessing Ki- $67^{+}$proliferating cells from transplanted hearts in recipients adoptively transferred with WT or $C c r 2^{4 C p t l a}$ monocytes revealed no significant

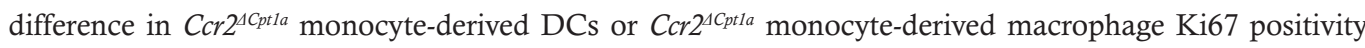
compared with WT counterparts (Supplemental Figure 6, B and C). Finally, we did not observe a difference in $\mathrm{CD} 45.2^{+}$cells in the transplanted hearts of HTx recipients adoptively transferred with either $\mathrm{Ccr} 2^{4 \text { Cptla }}$ monocytes or WT monocytes (Supplemental Figure 6D).

\section{Discussion}

In our current study, we demonstrate that pharmacological inhibition of FAO significantly prolongs heart allograft survival. Adoptive transfer experiments revealed that FAO inhibition results in a significant impairment in monocyte differentiation, which results in a reduction of monocyte-derived DCs and macrophages in the transplanted heart, with no impairment on the homing of monocytes to the heart allograft. In vitro experiments confirmed that FAO inhibits monocyte differentiation, while not directly affecting $\mathrm{T}$ cell reactivity. Eto did not extend allograft survival in $\mathrm{Ccr}^{-/-}$recipients, further supporting FAO-mediated dampening of alloimmunity through its effects on monocytes. Finally, genetic deletion of Cpt1a in monocytes mirrored effects on monocyte differentiation in heart allografts observed with 
A
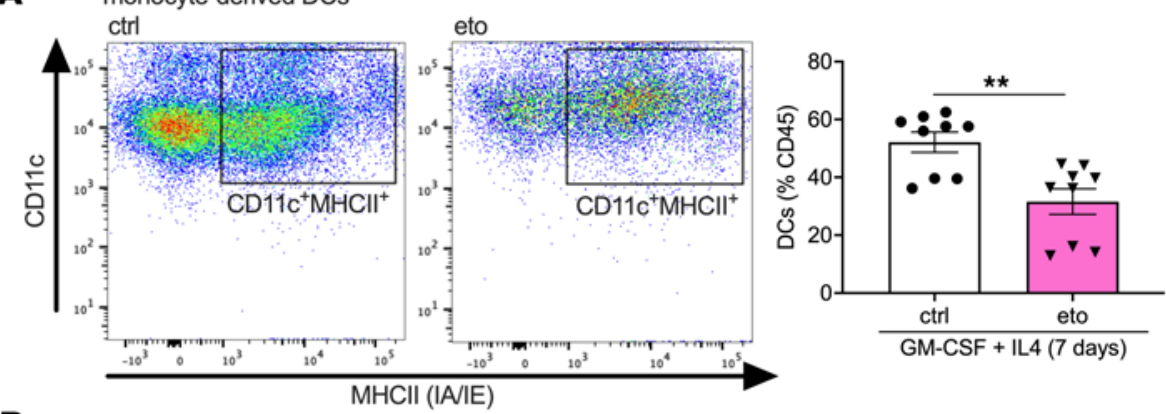

B
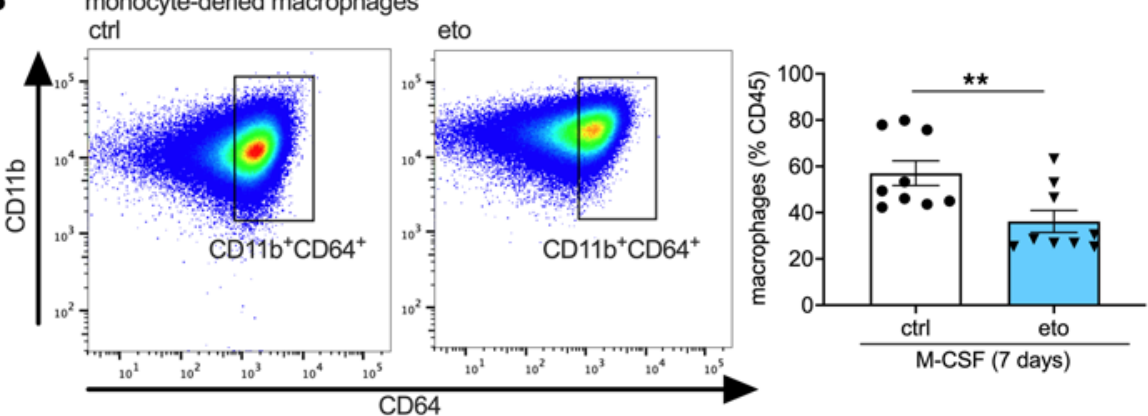

C

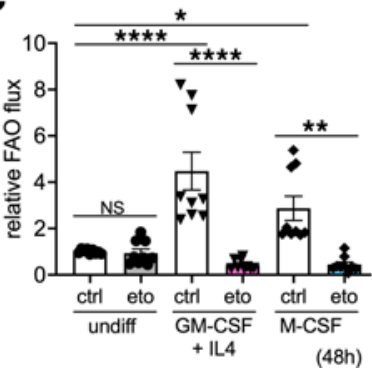

D

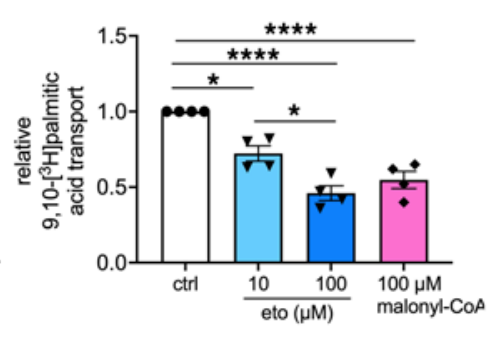

E

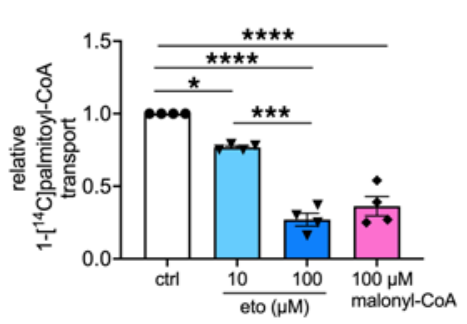

$\mathbf{F}$

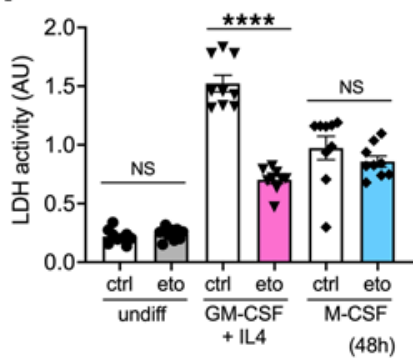

Figure 4. FAO inhibition impairs monocyte-to-DC and monocyte-to-macrophage differentiation in vitro. (A and B) Flow cytometric assessment of

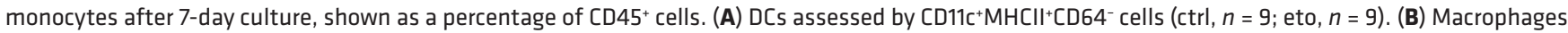
assessed by CD11 +CD64+CD24- cells (ctrl, $n=9$; eto, $n=9$ ). (C) FAO flux in monocytes in media only or treated for 48 hours with GM-CSF + IL4 (DC differentiation condition) or M-CSF (macrophage differentiation condition), as assessed by $\left[9,10-{ }^{3} \mathrm{H}\right]$ palmitic acid radioisotopic incorporation (ctrl, $n=9$; eto, $n=9$ ). (D and E) Cpt1 activity assay in intact mitochondria from monocytes treated with 10 or $100 \mu \mathrm{M}$ etomoxir or $100 \mu \mathrm{M}$ malonyl-CoA coincubated with either (D) 9,10-[3H]palmitic acid ( $n=4)$ or $(\mathbf{E}) 1-\left[{ }^{14} \mathrm{C}\right]$ palmitoyl-E $(n=4)$. (F) Cell death in monocytes in media only or treated for 48 hours with GM-CSF + IL4 or M-CSF, as assessed by lactate dehydrogenase (LDH) release (ctrl, $n=9$; eto, $n=9$ ). Data are shown as mean of individual data points from 3 independent experiments \pm SEM. ${ }^{*} P<0.05 ;{ }^{* *} P<0.01 ;{ }^{* * *} P<0.001 ;{ }^{* * *} P<0.0001$ by (A and $\left.\mathbf{B}\right) t$ test or ANOVA and (C-F) Bonferroni's post hoc test.

pharmacological inhibition of FAO. Collectively, these findings present a possibly previously unreported role for FAO in monocyte differentiation in the context of the regulation of alloimmunity.

Due to a reported role of glycolysis in macrophages and DCs, with sparse reports on specific oxidative metabolic pathways, we sought to investigate the role of FAO on heart allograft rejection. FAO has been reported to support an LPS-induced phenotype resembling the Warburg effect in glucose-deprived monocytes (23). However, this conclusion was primarily supported by results obtained by extracellular flux analysis. Raulien et al. reported that glutamine and fatty acids drive oxidative metabolism in the absence of glucose, and, for FAO, this occurs at the expense of lipid droplets (23). While this altered metabolic state in glucose deprivation is reported to provide sufficient energy to sustain functional properties such as cytokine secretion, migration, and phagocytosis, it does not prevent a rise in AMP/ATP ratio or decreased respiratory burst (23). In the context of aging, monocytes are key producers of inflammatory cytokines, and monocytes from aged individuals were found to contain high levels of lipid droplets and impaired FAO, in part, through the downregulation of peroxisome proliferator-activated receptor- $\alpha$ (29), supporting the importance of FAO in monocyte function.

It is important to note that glycolysis is important in monocyte functions, including their activation and adhesion (30), and also plays a role in modulating $\mathrm{CD} 8^{+} \mathrm{T}$ cell function in human Chagas disease (31) 
A

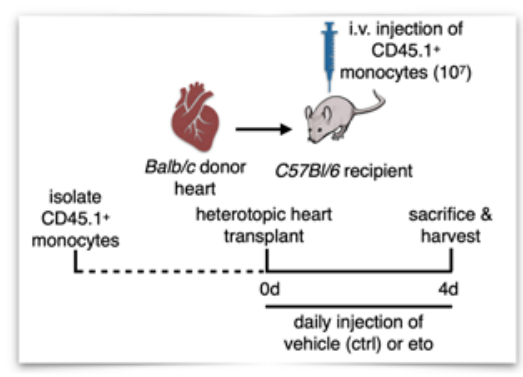

B

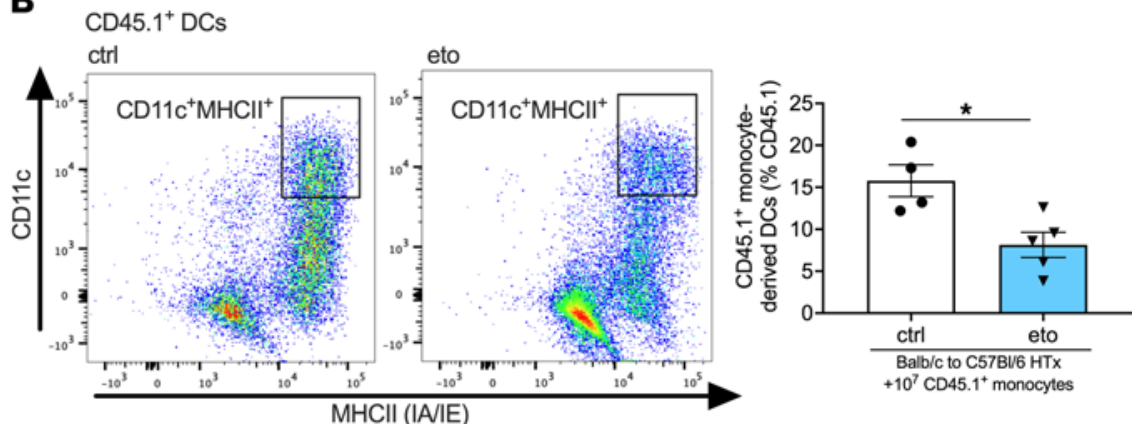

C $\quad \mathrm{CD} 45.1^{+}$macrophages
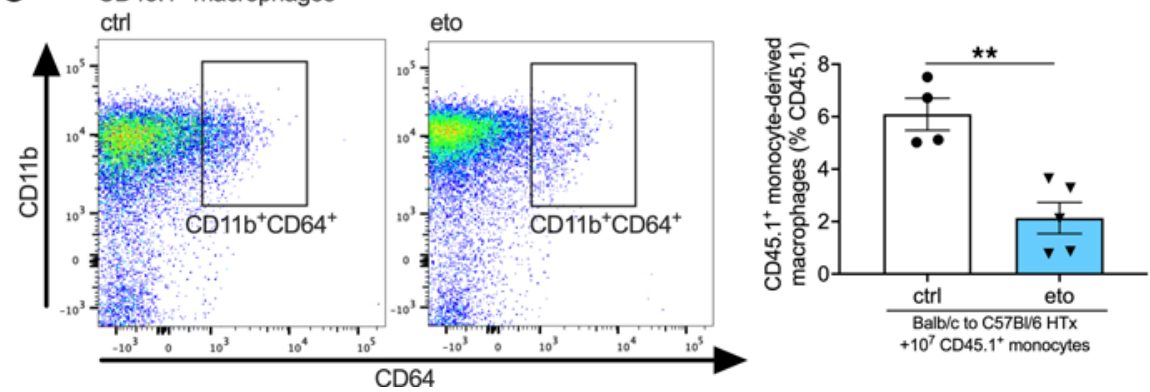

Figure 5. FAO inhibition impairs monocyte-derived DC and monocyte-derived macrophage differentiation in heart allografts in vivo. (A) Schematic representation of adoptive transfer of $10^{7} \mathrm{CD} 45.1^{+}$monocytes isolated from Pepboy mice intravenously injected into HTx recipients at the time of engraftment. (B and C) Flow cytometric assessment of BALB $\rightarrow$ B6 heart allografts 4 days after HTx that were adoptively transferred with CD45.1+ monocytes at the

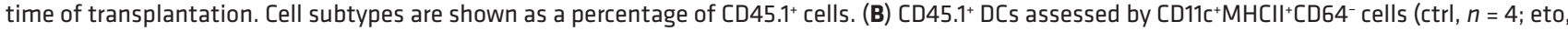
$n=5$ ). (C) CD45.1+ macrophages assessed by CD11b+CD64+CD24- cells (ctrl, $n=4$; eto, $n=5$ ). Data are shown as mean \pm SEM. ${ }^{*} P<0.05$; ${ }^{*} P<0.01$ by $t$ test.

and neutrophils in human hepatocellular carcinoma (32). Lee et al. demonstrated that LPS stimulation increases the rate of glycolysis in human classical monocytes and that 2-deoxyglucose-mediated inhibition of glycolysis impaired LPS-induced activation and adhesion of monocytes (30). In this setting, increased glycolysis was regulated by mTOR-induced glucose transporter 1 expression, which subsequently led to increased accumulation of reactive oxygen species and activation of p38 MAPK, resulting in subsequent activation and adhesion of monocytes. However, these studies did not explore a direct role for FAO in monocyte function, in particular, in the context of an in vivo allogeneic environment.

As with all pharmacological approaches, FAO inhibition by eto would be expected to also affect other cell types that may be reliant on FAO, including cardiomyocytes (33), skeletal muscle cells (34), endothelial cells (35-37), and renal tubular epithelial cells (38). Within immune cell subsets, FAO has also been reported to be important in macrophage polarization (39) and $\mathrm{CD} 8^{+} \mathrm{T}$ memory (Tmem) cells (40); however, recent evidence using genetic models has brought into question the specific requirement for Cpt1a or long-chain FAO in M2 macrophage polarization (41) and Tmem cells $(42,43)$. Consistent with reports that Cpt1a/FAO is dispensable in T cells $(42,43)$, we did not observe direct effects of pharmacological inhibition of FAO on T cell activation. Eto has been shown to affect antigen-stimulated IL-15 Tmem-like cells in vitro (44); however, genetic studies targeting FAO enzymes revealed no impairment in Tmem cell formation in vivo (42). In our work, we did not investigate Tmem cells; however, the abrogation of survival benefit and $\mathrm{T}$ cell changes in $\mathrm{Crr}^{-{ }^{-}}$recipient mice support the hypothesis of a FAO-dependent role in monocytes or monocyte-derived cells in our experiments. Administration of eto has been reported to improve cardiac function in fatty acid-induced ischemic injury (45) and left ventricular performance in pressure-overloaded hearts (46-48); and it has also been tested in limited clinical trials in patients with congestive heart failure, demonstrating improved ejection fraction and cardiac output $(49,50)$. In our studies, we did not observe any further heart allograft survival benefit in $\mathrm{Ccr}^{-1-} \mathrm{HTx}$, suggesting that the efficacy of eto is dependent on the presence of Ccr2-expressing cells in the recipient. Of note, the mouse model of heterotopic HTx results in a "nonfunctional" graft, where blood flows from the recipient aorta into the donor ascending aorta, supplying the coronary arteries. Blood then drains into the right atrium via the coronary sinus, which is then pumped into the right ventricle and, subsequently, enters the recipient inferior vena cava through the pulmonary artery. Coronary supply of arterial 
A

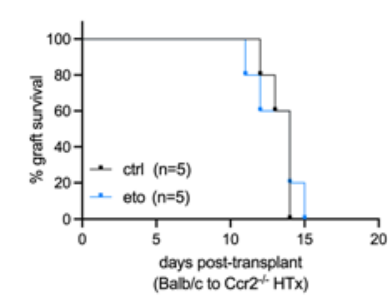

D
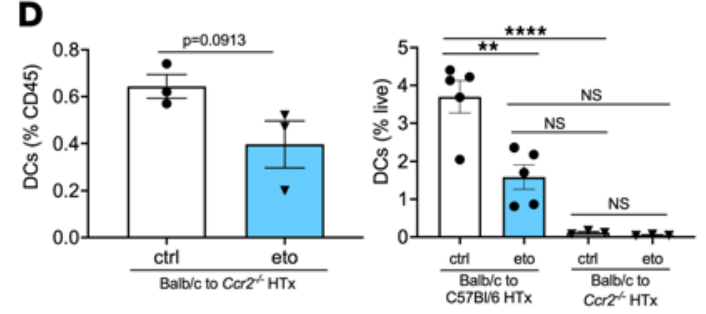

B
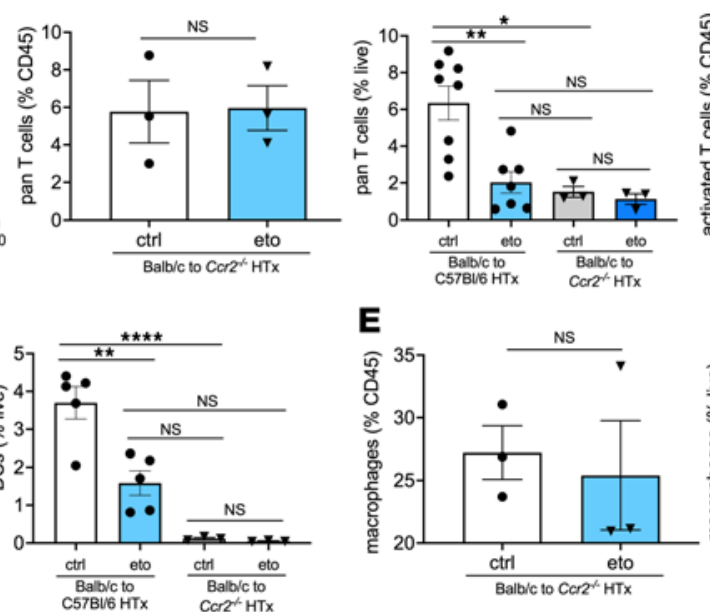

\section{C}
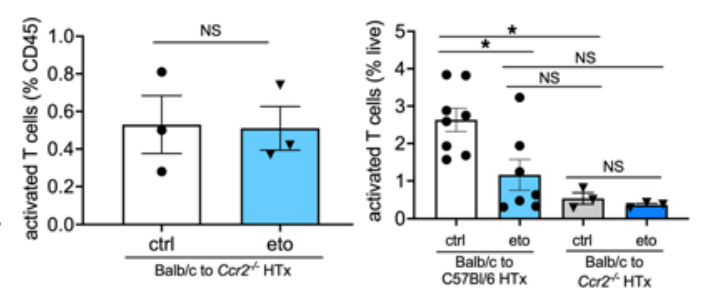

$\mathbf{F}$
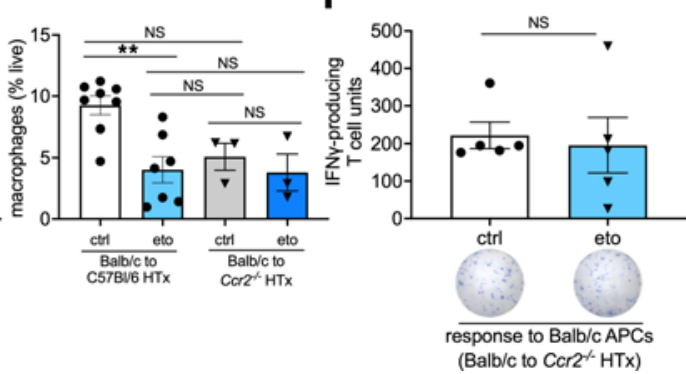

Figure 6. Loss of Ccr2 in HTx recipients abrogates the effects of FAO inhibition. (A) Comparison of graft survival in vehicle-treated (ctrl-treated) or etomoxir-treated (eto-treated) BALB $\rightarrow \mathrm{B} 6$ CCR2 ${ }^{-1-}$ heart allografts. There was no statistical difference in survival $(P=0.7698$ by Mantel-Cox test). (B-E) Flow cytometric assessment in BALB $\rightarrow$ C Cr2 ${ }^{-1-}$ heart allografts 4 days after transplant, shown as a percentage of CD45 $5^{+}$cells (left graph) or live cells (right graph; data for live cells from BALB $\rightarrow$ B6 HTx is replotted from Figures 1 and 2). (B) Pan T cells assessed by CD3+CD90.2 $2^{+}$cells (ctrl, $n=8$; eto, $n=7$ for BALB $\rightarrow$ B6 HTx; ctrl, $n=3$; eto, $n=3$ for BALB B6 Ccr2- $2^{--} \mathrm{HTX}$ ). (C) Activated T cells assessed by CD69+CD3+CD90.2 ${ }^{+}$cells (ctrl, $n=8$; eto, $n=7$ for BALB $\rightarrow$ B6 HTx; ctrl, $n=3$; eto, $n=3$ for BALB $\rightarrow$ B6 Ccr2 ${ }^{-1-} \mathrm{HTX}$ ). (D) DCs assessed by CD11c ${ }^{+}$MHCII+CD64 cells (ctrl, $n=5$; eto, $n=5$ for BALB $\rightarrow$ B6 HTx; ctrl, $n=3$; eto, $n=3$ for BALB B6 Ccr2 ${ }^{-/-} \mathrm{HTX}$ ). (E) Macrophages assessed by CD11 b+CD64+CD24- cells (ctrl, $n=8$; eto, $n=7$ for BALB $\rightarrow$ B6 HTx; ctrl, $n=3$; eto, $n=3$ for BALB $\rightarrow$ B6 C Cr2 ${ }^{-/-} \mathrm{HTX}$ ). (F) ELISPOT assay performed using splenocytes procured 4 days after transplantation from ctrl or eto-treated BALB B6 Ccr2 ${ }^{-1-}$ heart allografts. Samples were stimulated with donor-derived (BALB) APCs and assessed for the production of IFN $-\gamma(c t r l, n=5$; eto, $n=5)$. Data are shown as mean \pm SEM. ${ }^{*} P<0.05 ;{ }^{* *} P<0.01$; ${ }^{* * *} \mathrm{P}<0.0001$ ( $\mathrm{B}-\mathrm{E}$, left graphs, and $\mathrm{F}$ ) by t test or (B-E, right graphs) ANOVA and Bonferroni's post hoc test.

blood reestablishes sinus rhythm in the transplanted heart after reperfusion; however, the left chambers of the heart remain pressure underloaded (51).

One limitation of the current study is our use of eto as a pharmacological inhibitor of FAO. Eto has been reported to have off-target effects on oxidative metabolism depending on dose and cell type $(52,53)$. We used this drug because of our extensive previous experience with its pharmacokinetics and pharmacodynamics in multiple cell types both in vitro and in vivo $(35-37,52,53)$. By using a dose of $100 \mu \mathrm{M}$ in vitro, we aimed to avoid some of the off-target effects on complex I in the electron transport chain or coenzyme A homeostasis that have been described at doses above $100 \mu \mathrm{M}$ (specifically $200 \mu \mathrm{M}$ and above) $(41-43,54)$. We further confirmed that eto was able to inhibit FAO flux and $\mathrm{Cpt} 1$ activity at multiple doses in murine monocytes. In our in vitro model of monocyte differentiation, we were able to demonstrate a significant reduction in FAO upon treatment with eto; however, in vivo assessment of metabolic fluxes remains challenging. Off-target effects of eto, and the doses which elicit them, are harder to investigate. However, our experiments using $\mathrm{CCr}^{-1-}$ recipient mice demonstrated abrogation of the effect of pharmacological inhibition of FAO and support our conclusion regarding the importance of FAO in monocyte differentiation. In addition, the role of FAO in monocyte differentiation in the context of HTx in vivo was further verified using conditional deletion of Cpt1a in monocytes. The mechanism whereby FAO regulates monocyte differentiation remains to be determined. Our observation that both pharmacological inhibition of $\mathrm{Cpt} 1$ as well as genetic deletion of Cpt1a can reduce monocyte-to-macrophage and monocyte-to-DC differentiation in heart allografts supports a role for FAO in these functions. Furthermore, investigation into the specific roles of FAO in DCs and macrophages in allograft rejection is required. In addition to promoting alloimmunity and allograft rejection, monocytic populations may also play an important role in limiting alloimmune responses. It has previously been demonstrated that $\mathrm{CD} 11 \mathrm{~b}^{+} \mathrm{CD} 115^{+} \mathrm{Ly} 6 \mathrm{C}^{+}$monocytes are required to maintain heart transplant tolerance induced by donor splenocyte transfusion in addition to anti-CD40L monoclonal antibody (55). Early after transplantation, monocytes migrate from the bone marrow to the transplanted organ, where they can prevent the initiation of adaptive immune responses that lead to allograft rejection and participate in the development of Tregs. It has been reported that reducing monocyte-derived macrophages impairs transplant tolerance, in part, through increasing both $\mathrm{CD} 8^{+} \mathrm{T}$ cell numbers and reducing Treg expansion 
A

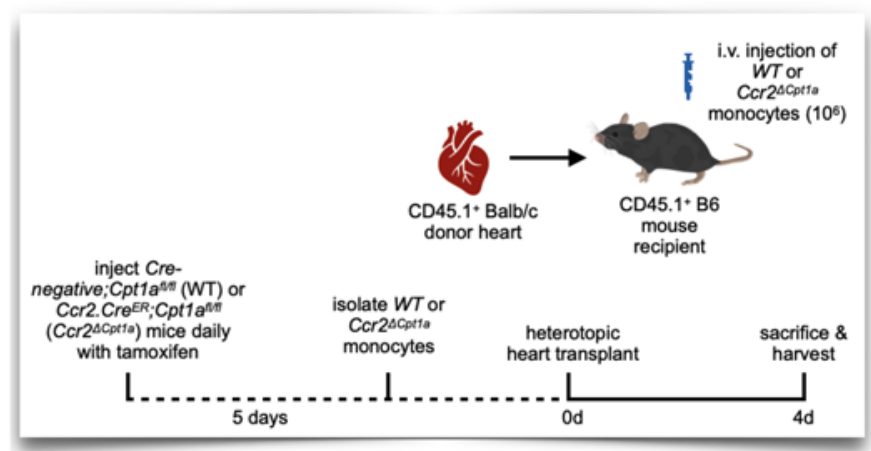

B $\quad$ CD $45.2^{+}$monocyte-derived DCs
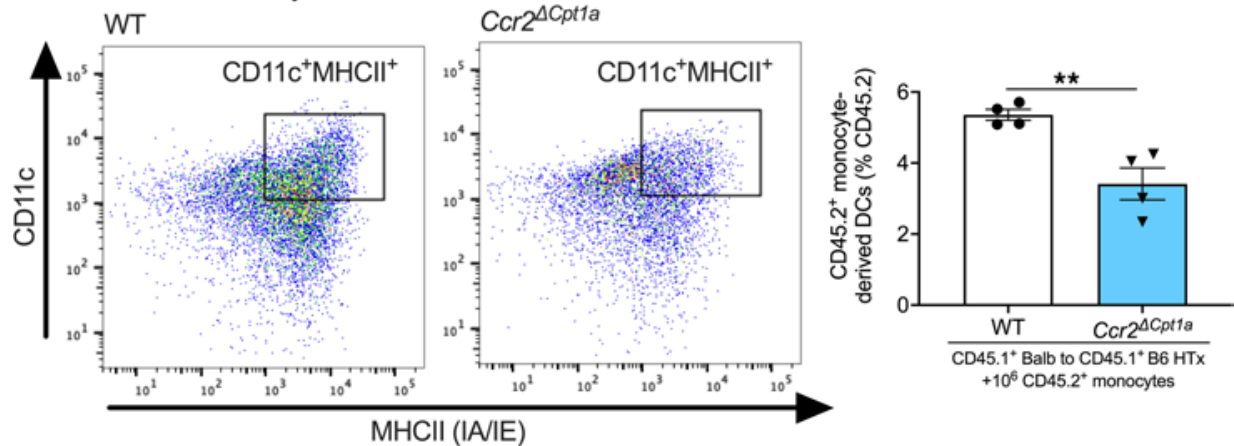

C $\quad$ CD45.2 $2^{+}$monocyte-derived macrophages
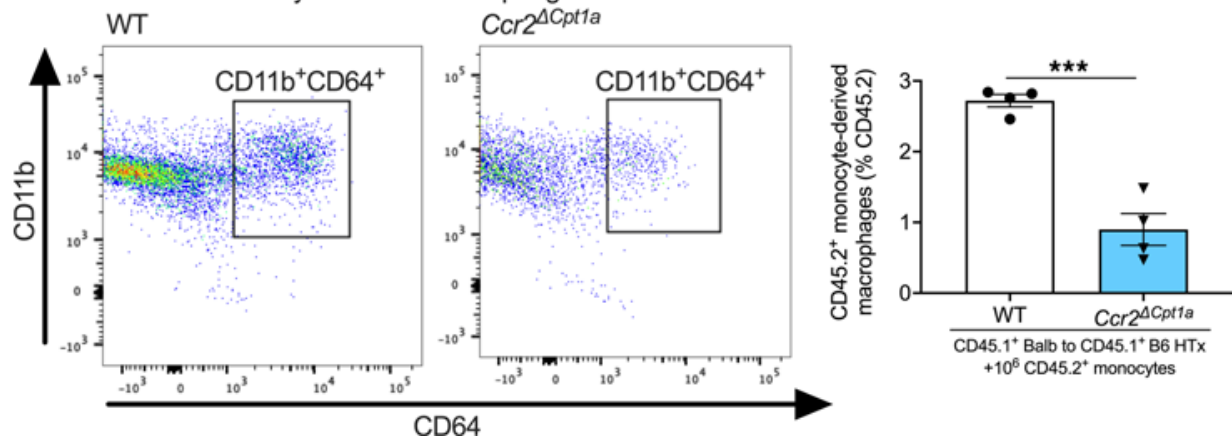

Figure 7. Genetic deletion of Cpt1a monocyte-derived DC and monocyte-derived macrophage differentiation in heart allografts in vivo. (A) Schematic representation of adoptive transfer of $10^{6}$ monocytes isolated from Cre- $\mathrm{Cpt1a}^{\mathrm{fl} / \mathrm{fl}}$

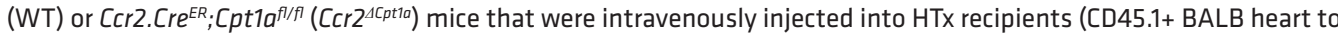
CD45.1+ B6 recipient) at the time of engraftment. (B and C) Flow cytometric assessment of transplanted hearts from $\mathrm{CD} 45.1^{+}$BALB $\rightarrow$ CD45.1 $1^{+}$B6 allografts 4 days after HTx, adoptively transferred with WT or Ccr2 ${ }^{4 \text { Cptla }}$ monocytes. All cell subtypes are shown as a percentage of CD45.2+ cells. (B) CD45.2+ DCs assessed by CD11c ${ }^{+} \mathrm{MHClI}^{+} \mathrm{CD} 64^{-}$cells (WT, $\left.n=4 ; C c r 2^{4 C p t 1 a}, n=4\right)$. (C) CD45.2 $2^{+}$macrophages assessed by CD11 ${ }^{+} C D 64^{+}{ }^{+} C D 24^{-}$cells $\left(W T, n=4 ; C c r 2^{4 C p t 1 a}, n=4\right)$. Data are shown as mean $\pm \mathrm{SEM}$. ${ }^{* *} P<0.01 ;{ }^{* *} P<0.001$ by $t$ test.

$(56,57)$, suggesting that the role monocytes play in allogeneic immune responses may vary between acute and tolerogenic settings. As our work demonstrates that inhibition of FAO can impair the differentiation of monocyte-derived cells in acute HTx rejection, it would be of interest to further explore the role of FAO in monocyte dynamics in tolerogenic settings. The potential clinical utilization of eto is unlikely, as clinical trials for eto were halted in phase II due to unacceptably high liver transaminase levels and associated hepatotoxicity $(58,59)$. Nevertheless, our study highlights the potential clinical utility of other pharmacological inhibitors of FAO, which warrants further investigation.

\section{Methods}

Animals. Six- to 10-week-old BALB (no. 000651), B6 (no. 000664), B6.SJL-Ptprc Pepc $c^{b}$ BoyJ (known as Pepboy, CD45.1+ B6 background; no. 002014), CByJ.SJL(B6)-Ptprc ${ }^{a} / J$ (CD45.1+ BALB background; no. 006584), B6.129S4-Ccr2 $2^{\text {tmllfc }} / J\left(C c r 2^{-/-}\right.$; no. 004999) and B6(Cg)-Cpt1atm1c(EUCOMM)Hmgu/RjnsJ(Cpt1a $a^{f / f l}$; 
refs. 36, 42; no. 032778) mice were purchased from The Jackson Laboratory. Ccr2.Cre ${ }^{E R}$ transgenic mice were a gift from Andrew Gelman (Washington University School of Medicine). Ccr2.Cre ${ }^{E R}$ mice were intercrossed with $C p t 1 a^{f l f l}$ mice to generate $C c r 2^{4 C p t a a}$ mice. Littermate $C_{r e} C_{p t 1} a^{f l f l}$ mice were used as ctrls (referred to herein as WT). Animals were bred and housed under standard conditions.

Reagents. Fluorochrome-conjugated antibodies used in this study are listed in Supplemental Table 1. The LIVE/DEAD fixable violet dead cell stain kit was from Thermo Fisher Scientific. Brilliant Stain Buffer Plus 1000t and BD Horizon Fixable Viability Stain 510 were from BD Biosciences. Concanavalin A, sodium hydroxide, trichloroacetic acid (TCA), (+)-eto sodium salt hydrate, tamoxifen, and other standard laboratory chemicals were from MilliporeSigma.

Heterotopic heart transplant. Allogeneic (BALB $\rightarrow$ B6) and syngeneic (B6 $\rightarrow$ B6) heterotopic HTx was performed as previously described $(60,61)$. Anesthesia was induced by a mixture of ketamine ( $80-100 \mathrm{mg} / \mathrm{kg}$ ) and xylazine $\mathrm{HCl}(8-12 \mathrm{mg} / \mathrm{kg})$, administered i.p., and maintained with $1 \%-2 \%$ isoflurane gas, as required. Briefly, donor hearts were implanted into the abdominal cavity of recipient mice, where the donor ascending aorta and pulmonary artery were anastomosed to the recipient infrarenal aorta and inferior vena cava, respectively. Donor hearts were maintained on ice between procurement and implantation, and cold ischemic times were less than 60 minutes. Heart transplant recipients were administered either vehicle $\left(\mathrm{H}_{2} \mathrm{O}\right)$ or eto $(20 \mathrm{mg} / \mathrm{kg})$ daily by i.p. injection. Function of transplanted hearts was assessed by daily direct palpation along the abdomen adjacent to the implantation site until cessation of palpable heartbeat. Heart graft function was assessed using the following scale: +++ , soft graft with strong contraction; ++ , mild turgor and mild contraction; + , hard turgor and weak contraction; -, no palpable contraction. Cessation of palpable heartbeat was verified at time of sacrifice by laparotomy. For experiments involving $\mathrm{Ccr}^{-/-}$mice, BALB donor hearts were transplanted into $\mathrm{C} c 2^{-1-}$ recipient mice. For experiments involving adoptive transfer of WT or Ccr2 $2^{4 \text { Cptla }}$ monocytes, CD $45.1^{+}$ BALB donor hearts were transplanted into CD $45.1^{+} \mathrm{B} 6$ recipient mice.

Monocyte isolation, adoptive transfer of monocytes, and in vitro differentiation. Bone marrow-derived monocytes were obtained from long bones using the MACS Monocyte Isolation Kit (Miltenyi Biotec) following the manufacturer's protocol. For adoptive transfer experiments, $10^{7} \mathrm{~B} 6 \mathrm{CD} 45.1$, monocytes were intravenously injected at time of HTx (62). For monocyte differentiation assays, $10^{6}$ monocytes were cultured in RPMI complete media (Invitrogen) supplemented with 10\% FBS, penicillin/streptomycin (Invitrogen; 120 units $/ \mathrm{mL}$ and $100 \mu \mathrm{g} / \mathrm{mL}$, respectively) and $0.05 \mathrm{mM}$ 2-mercaptoethanol. Stimulation of monocytes to differentiate toward a DC or macrophage lineage was achieved by culturing with GM-CSF ( $25 \mathrm{ng} / \mathrm{mL}$, Miltenyi Biotec) + IL-4 (25 ng/mL, Miltenyi Biotec) or M-CSF (100 ng/mL, Miltenyi Biotec), respectively. Monocytes were treated with $100 \mu \mathrm{M}$ eto for indicated experiments.

Flow cytometry. Spleen and mediastinal lymph nodes were procured and rinsed with PBS. Then, the tissue was minced and digested with washing with $0.5 \mathrm{mg} / \mathrm{mL}$ collagenase I (MilliporeSigma) +60 units $/ \mathrm{mL}$ DNase I (MilliporeSigma) in DMEM, centrifuged, and filtered through a $70 \mu \mathrm{m}$ filter. Then, RBC lysis buffer (MilliporeSigma) was used, followed by a final centrifugation step and freezing using CTL-Cryo ABC Freezing Kit (Cellular Technology Limited [CTL]). Hearts were procured, perfused with cold PBS, and then minced and digested with 450 units $/ \mathrm{mL}$ collagenase I (MilliporeSigma) + 60 units hyaluronidase type 1-s (MilliporeSigma) +60 units/mL Dnase I in DMEM. The tissue in digestion solution was then centrifuged and filtered through a $40 \mu \mathrm{m}$ filter, followed by RBC lysis and a final centrifugation step and freezing using CTL-Cryo ABC Freezing Kit (CTL). Flow cytometry was performed as previously described (63) using either a BD LSR Fortessa or a BD X-20 BD (BD Biosciences). Representative flow plots in the figures illustrate the distribution of the target population, which is presented in the associated graph(s) relative to $\mathrm{CD} 45^{+}$or live cells, unless otherwise indicated. Flow cytometry data were analyzed by FlowJo software (version 10.71).

For identification of total T cells, we used the following gating strategy: FSC/SSC, single live cells, $\mathrm{CD} 5^{+}, \mathrm{CD}^{+} \mathrm{CD} 90.2^{+}, \mathrm{CD}^{+} 9^{+}, \mathrm{CD}^{+}, \mathrm{CD}^{+}$, and $\mathrm{Foxp}^{+} \mathrm{CD}^{+}$populations were identified from total $\mathrm{T}$ cell pools. For identification of DCs, we used the following gating strategy: FSC/SSC, live cells, CD45 and $\mathrm{CD} 11 \mathrm{c}^{+} \mathrm{MHCII}{ }^{+}$. For identification of macrophages, we used the following gating strategy: FSC/SSC, single live cells, $\mathrm{CD} 45^{+}, \mathrm{CD} 24^{-}$, and $\mathrm{CD} 11 \mathrm{~b}^{+} \mathrm{CD} 64^{+}$.

For adoptive transfer experiments, the following gating strategy was used for CD $45.1^{+} \mathrm{DCs}$ : FSC/SSC, single live cells, $\mathrm{CD} 45^{+}, \mathrm{CD} 45.1^{+}, \mathrm{CD} 64^{-}$, and $\mathrm{CD} 11 \mathrm{c}^{+} \mathrm{MHCII}{ }^{+}$. The following gating strategy was used for macrophages: FSC/SSC, single live cells, CD $45^{+}, \mathrm{CD} 45.1^{+}, \mathrm{CD} 24^{-}$, and $\mathrm{CD} 64^{+} \mathrm{CD} 11 \mathrm{~b}^{+}$. Similar gating strategies were used for the assessment of in vitro monocyte differentiation. 
For experiments involving adoptive transfer of WT or $C \mathrm{cr} 2^{4 \mathrm{Cptla}}$ monocytes, the following gating strategy was used for CD45.2 $2^{+}$DCs: FSC/SSC, single live cells, CD45 ${ }^{+}, \mathrm{CD} 45.2^{+}, \mathrm{CD}^{-}$, and $\mathrm{CD} 11 \mathrm{c}^{+} \mathrm{MHCII}^{+}$. The following gating strategy was used for CD $45.2^{+}$macrophages: FSC/SSC, single live cells, $\mathrm{CD}^{+} 5^{+}$, CD45.2 $2^{+}, \mathrm{CD}_{24}^{-}$, and CD11b ${ }^{+} \mathrm{CD} 64^{+}$.

ELISPOT. Precoated strips with antibodies directed against mouse IFN- $\gamma$, TNF- $\alpha$, or IL-2 were purchased from CTL. Splenocytes were obtained from HTx recipients, rinsed with PBS, and then the tissue was minced and digested with $0.5 \mathrm{mg} / \mathrm{mL}$ collagenase I (MilliporeSigma) + 60 units $/ \mathrm{mL}$ DNase I (MilliporeSigma) in DMEM, centrifuged, and filtered through a $70 \mu \mathrm{m}$ filter. Then, RBC lysis buffer (MilliporeSigma) was used, followed by a final centrifugation step and freezing using the CTL-Cryo ABC Freezing Kit (CTL). Media only and $5 \mu \mathrm{g} / \mathrm{mL}$ Concanavalin A (MilliporeSigma) were used as negative and positive ctrls, respectively. Isolated splenocytes from BALB or B6 mice were irradiated with 20 Gy using an X-RAD 320 Biological Irradiator (Precision X-Ray) and used as "activated" APCs. Splenocytes (100,000 cells) were coincubated with positive ctrl, negative ctrl, or activated APCs (100,000 cells) and cultured in CTL test serum-free media supplemented with penicillin/streptomycin (Invitrogen; 120 units/mL and 100 $\mu \mathrm{g} / \mathrm{mL}$, respectively) and $2 \mathrm{mM}$ L-glutamine (Invitrogen). 2-3 technical replicate wells were used for each condition, and $n$ numbers represent samples from individual HTx. Single-color ELISPOT assays were performed following the manufacturer's specifications, and plates were dried, scanned, and analyzed using an Immunospot S6 Universal Analyzer (CTL).

Histology. Tissues were procured and fixed in 4\% paraformaldehyde before embedding in OCT for cryosections or processed for paraffin embedding. $5 \mu \mathrm{m}$ sections were stained with hematoxylin and eosin to assess histopathological changes.

Radioisotope tracer experiments. 50,000 cells per well were seeded and allowed to attach before gentle washing and incubation for 48 hours in media containing $0.4 \mu \mathrm{Ci} / \mathrm{mL}$ (24.24 $\mathrm{nM}$ final concentration) $\left[5-{ }^{3} \mathrm{H}\right]$ glucose (Perkin Elmer) or $2 \mu \mathrm{Ci} / \mathrm{mL}$ (66.67 nM final concentration) $\left[9,10-{ }^{3} \mathrm{H}\right]$ palmitate (Perkin Elmer) for assessment of glycolytic or FAO flux, respectively. For FAO flux assays, $50 \mu \mathrm{M}$ L-carnitine solution (MilliporeSigma) was supplemented to the media. To assess the production of ${ }^{3} \mathrm{H}_{2} \mathrm{O}$ after a cumulative 48 hours of drug treatment, media was transferred to glass vials and sealed with rubber stoppers. ${ }^{3} \mathrm{H}_{2} \mathrm{O}$ was captured in hanging wells within the glass vials containing Whatman filter paper soaked with equivolume $\mathrm{H}_{2} \mathrm{O}$ over a period of 48 hours at $37^{\circ} \mathrm{C}$ to reach saturation. Radioactivity for radioisotope tracer studies was determined by liquid scintillation counting using a Beckman Coulter LS6500 scintillation counter.

Cpt1 activity assay. Cpt1 activity was performed on isolated mitochondria from murine monocytes. Briefly, bone marrow-derived monocytes were obtained from long bones using the MACS Monocyte Isolation Kit (Miltenyi Biotec) following the manufacturer's protocol, and then mitochondria were isolated using a mitochondria isolation kit (Thermo Fisher Scientific). Isolated mitochondria were coincubated with radioisotope-labeled 9,10-[ $\left[{ }^{3} \mathrm{H}\right]$ palmitic and $1-\left[{ }^{14} \mathrm{C}\right]$ palmitoyl-CoA in an assay solution containing $20 \mathrm{mM}$ HEPES, $75 \mathrm{mM} \mathrm{KCl}, 2 \mathrm{mM} \mathrm{KCN}, 1 \%$ fatty acid-free BSA, and $0.25 \mathrm{mM}$ L-carnitine, adapted from previous publications (64-66). After incubating for 3 minutes at $37^{\circ} \mathrm{C}$, the reaction was quenched with $3 \mathrm{M}$ TCA, spun down at 12,000 $\mathrm{g}$ for 5 minutes, and then washed with $2 \mathrm{mM}$ TCA and centrifuged again, before removing the supernatant and extracting the mitochondrial pellet for liquid scintillation counting using a Beckman Coulter LS6500 scintillation counter. The endogenous Cpt1 inhibitor malonyl-CoA (MilliporeSigma) was used as a positive ctrl.

Proliferation assay. Thymidine incorporation assay was used to assess cell proliferation. Briefly, 10,000 cells per well were seeded and incubated with $1 \mu \mathrm{Ci} / \mathrm{mL}\left[{ }^{3} \mathrm{H}\right]$ thymidine (Perkin Elmer) for specified time periods. Cells were fixed with $100 \%$ ethanol for 15 minutes at $4^{\circ} \mathrm{C}$ and then precipitated with 10\% TCA (MilliporeSigma) and lysed with $0.1 \mathrm{~N} \mathrm{NaOH}$ (MilliporeSigma). The amount of $\left[{ }^{3} \mathrm{H}\right]$ thymidine incorporated into DNA was measured by liquid scintillation counting. T cells were isolated from the spleens of HTx recipients using the pan T cell isolation kit (Miltenyi Biotec) and then cultured in RPMI (Invitrogen) supplemented with 10\% FBS, penicillin/streptomycin (Invitrogen; 120 units/ $\mathrm{mL}$ and $100 \mu \mathrm{g} / \mathrm{mL}$, respectively), $0.05 \mathrm{mM}$ 2-mercaptoethanol, and 20 units $/ \mathrm{mL}$ recombinant IL-2 (Miltenyi Biotec). Monocytes were cultured as indicated above. T cells or monocytes were treated with $100 \mu \mathrm{M}$ eto for indicated experiments.

Cytotoxicity assessment. The Cytotoxicity Detection Kit (Roche CustomBiotech), which quantifies cytotoxicity/cytolysis based on the measurement of $\mathrm{LDH}$ release from damaged cells, was used to assess the effects of eto on monocyte viability, as per the manufacturer's protocol. 
Statistics. All data are expressed as mean \pm SEM, unless otherwise indicated. Two-tailed Student's $t$ test was used for 2-group comparisons. For multiple group analysis, 1-way ANOVA with Bonferroni's post hoc test was used to calculate statistical significance. Log-rank (Mantel-Cox) test was used for survival curve comparison. $P$ values of less than 0.05 were considered statistically significant. Statistical analysis and graphics were performed using GraphPad Prism software and Microsoft Excel.

Study approval. Animal experiments were conducted in accordance with an approved Washington University School of Medicine Institutional Animal Care and Use Committee protocol (no. 20190173; no. 20190174).

\section{Author contributions}

AEG, DK, and BWW designed the research studies. YZ, HD, LY, YT, and BWW performed the experiments and data acquisition. YZ and BWW analyzed the data. LPS, GJP, DK, and AEG assisted in interpretation of the data and critically reviewed the manuscript. BWW wrote the manuscript. The order of the co-first authors was decided as follows: YZ performed the flow cytometry, ELISPOT, and in vitro experiments, and HD established the heterotopic heart transplant protocol in mice in our laboratory and performed all the microsurgeries for this study, monitored transplanted heart function and health of animals throughout the course of experiments, administered drugs daily, and harvested all tissues for downstream analysis. All authors read and discussed manuscript drafts.

\section{Acknowledgments}

This work was supported by a grant from the American Society of Transplantation Research Network (to BWW) and by the Joel D. Cooper Career Development Award from the International Society for Heart and Lung Transplantation (to BWW). This work was also supported by grants from the NIH (1P01AI116501, to DK and AEG; R01HL094601, to DK; R01HL151078, to DK, R01HL094601, to AEG), a Veterans Administration Merit Review grant (1I01BX002730, to DK), and The Foundation for Barnes-Jewish Hospital (to DK). This work was also supported by the Washington University Institute of Clinical and Translational Sciences, which is, in part, supported by the NIH/National Center for Advancing Translational Sciences Clinical and Translational Science Award grant UL1 TR002345. We acknowledge the Digestive Disease Research Core Center for assistance with histology, the Bursky Center for Human Immunology \& Immunotherapy Programs Core Laboratory for assistance with ELISPOT assays, and the Flow Cytometry \& Fluorescence Activated Cell Sorting Core in the Department of Pathology \& Immunology and the Siteman Flow Cytometry Core at the Washington University School of Medicine for assistance with flow cytometry.

Address correspondence to: Brian W. Wong, Department of Surgery, Washington University School of Medicine, 660 South Euclid Avenue, MSC 8234-0020-03, St. Louis, Missouri 63110, USA. Phone: 314.747.7316; Email: brian.wong@wustl.edu.

1. Alba AC, et al. Complications after heart transplantation: hope for the best, but prepare for the worst. Int J Transplant Res Med 2016;2:e22.

2. Lund LH, et al. The registry of the International Society for Heart and Lung Transplantation: thirty-fourth adult heart transplantation report-2017; focus theme: allograft ischemic time. J Heart Lung Transplant. 2017;36(10):1037-1046.

3. Gerhardt T, Ley K. Monocyte trafficking across the vessel wall. Cardiovasc Res. 2015;107(3):321-330.

4. Helft J, et al. GM-CSF mouse bone marrow cultures comprise a heterogeneous population of CD11c(+)MHCII(+) macrophages and dendritic cells. Immunity. 2015;42(6):1197-1211.

5. Lacey DC, et al. Defining GM-CSF- and macrophage-CSF-dependent macrophage responses by in vitro models. J Immunol. 2012;188(11):5752-5765.

6. Hamidzadeh K, et al. The transition of M-CSF-derived human macrophages to a growth-promoting phenotype. Blood $A d v$. 2020;4(21):5460-5472.

7. Italiani P, Boraschi D. From monocytes to M1/M2 macrophages: phenotypical vs. functional differentiation. Front Immunol. 2014;5:514.

8. Girlanda R, et al. Monocyte infiltration and kidney allograft dysfunction during acute rejection. Am J Transplant. 2008;8(3):600-607.

9. Zhang PL, et al. Monocyte-mediated acute renal rejection after combined treatment with preoperative Campath-1H (alemtuzumab) and postoperative immunosuppression. Ann Clin Lab Sci. 2004;34(2):209-213.

10. Vereyken EJ, et al. A shift towards pro-inflammatory CD16+ monocyte subsets with preserved cytokine production potential after kidney transplantation. PLoS One. 2013;8(7):e70152.

11. Van den Bosch TP, et al. Targeting the monocyte-macrophage lineage in solid organ transplantation. Front Immunol. 2017;8:153. 
12. Kopecky BJ, et al. Role of donor macrophages after heart and lung transplantation. Am J Transplant. 2020;20(5):1225-1235.

13. Van den Bosch TPP, et al. Pretransplant numbers of $\mathrm{CD}_{16}{ }^{+}$monocytes as a novel biomarker to predict acute rejection after kidney transplantation: a pilot study. Am J Transplant. 2017;17(10):2659-2667.

14. Chow KV, et al. Monocyte-derived dendritic cells impair early graft function following allogeneic islet transplantation. Cell Transplant. 2017;26(2):319-326.

15. Schroth S, et al. Innate functions of dendritic cell subsets in cardiac allograft tolerance. Front Immunol. 2020;11:869.

16. Ghesquiere B, et al. Metabolism of stromal and immune cells in health and disease. Nature. 2014;511(7508):167-176.

17. O'Neill LA, Pearce EJ. Immunometabolism governs dendritic cell and macrophage function. J Exp Med. 2016;213(1):15-23.

18. Du X, et al. Emerging roles of cellular metabolism in regulating dendritic cell subsets and function. Front Cell Dev Biol. $2018 ; 6: 152$.

19. Sadiku P, Walmsley SR. Hypoxia and the regulation of myeloid cell metabolic imprinting: consequences for the inflammatory response. EMBO Rep. 2019;20(5):e47388.

20. Wculek SK, et al. Metabolic control of dendritic cell functions: digesting information. Front Immunol. 2019;10:775.

21. Mazumdar C, et al. The untapped opportunity and challenge of immunometabolism: a new paradigm for drug discovery. Cell Metab. 2020;31(1):26-34.

22. Tabas I, Bornfeldt KE. Intracellular and intercellular aspects of macrophage immunometabolism in atherosclerosis. Circ Res. 2020;126(9):1209-1227.

23. Raulien N, et al. Fatty acid oxidation compensates for lipopolysaccharide-induced Warburg effect in glucose-deprived monocytes. Front Immunol. 2017;8:609.

24. Serbina NV, Pamer EG. Monocyte emigration from bone marrow during bacterial infection requires signals mediated by chemokine receptor CCR2. Nat Immunol. 2006;7(3):311-317.

25. Tsou CL, et al. Critical roles for CCR2 and MCP-3 in monocyte mobilization from bone marrow and recruitment to inflammatory sites. J Clin Invest. 2007;117(4):902-909.

26. Gelman AE, et al. CCR2 regulates monocyte recruitment as well as CD4 T1 allorecognition after lung transplantation. Am J Transplant. 2010;10(5):1189-1199.

27. Abdi R, et al. Differential role of CCR2 in islet and heart allograft rejection: tissue specificity of chemokine/chemokine receptor function in vivo. J Immunol. 2004;172(2):767-775.

28. Smith RN, et al. Chemokines and chronic heart allograft rejection. Transplantation. 2007;84(3):442-444.

29. Wang M, et al. Programmed PPAR- $\alpha$ downregulation induces inflammaging by suppressing fatty acid catabolism in monocytes iScience. 2021;24(7):102766.

30. Lee MKS, et al. Glycolysis is required for LPS-induced activation and adhesion of human CD14 CD16 monocytes. Front Immunol. 2019;10:2054.

31. Sanmarco LM, et al. Monocyte glycolysis determines CD8+ T cell functionality in human Chagas disease. JCI Insight. 2019;4(18):123490

32. Peng ZP, et al. Glycolytic activation of monocytes regulates the accumulation and function of neutrophils in human hepatocellular carcinoma. J Hepatol. 2020;73(4):906-917.

33. Lopaschuk GD, et al. Myocardial fatty acid metabolism in health and disease. Physiol Rev. 2010;90(1):207-258.

34. Kiens B. Skeletal muscle lipid metabolism in exercise and insulin resistance. Physiol Rev. 2006;86(1):205-243.

35. Kalucka J, et al. Quiescent endothelial cells upregulate fatty acid $\beta$-oxidation for vasculoprotection via Redox homeostasis Cell Metab. 2018;28(6):881-894.

36. Schoors S, et al. Fatty acid carbon is essential for dNTP synthesis in endothelial cells. Nature. 2015;520(7546):192-197.

37. Wong BW, et al. The role of fatty acid $\beta$-oxidation in lymphangiogenesis. Nature. 2017;542(7639):49-54.

38. Kang HM, et al. Defective fatty acid oxidation in renal tubular epithelial cells has a key role in kidney fibrosis development. Nat Med. 2015;21(1):37-46.

39. Huang SC, et al. Cell-intrinsic lysosomal lipolysis is essential for alternative activation of macrophages. Nat Immunol. 2014;15(9):846-855.

40. Pearce EL, et al. Enhancing CD8 T-cell memory by modulating fatty acid metabolism. Nature. 2009;460(7251):103-107.

41. Nomura M, et al. Fatty acid oxidation in macrophage polarization. Nat Immunol. 2016;17(3):216-217.

42. Raud B, et al. Etomoxir actions on regulatory and memory T cells are independent of Cpt1a-mediated fatty acid oxidation. Cell Metab. 2018;28(3):504-515.

43. Raud B, et al. Fatty acid metabolism in $\mathrm{CD}^{+} \mathrm{T}$ cell memory: Challenging current concepts. Immunol Rev. 2018;283(1):213-231.

44. O'Sullivan D, et al. Memory CD8(+) T cells use cell-intrinsic lipolysis to support the metabolic programming necessary for development. Immunity. 2014;41(1):75-88.

45. Lopaschuk GD, et al. Etomoxir, a carnitine palmitoyltransferase I inhibitor, protects hearts from fatty acid-induced ischemic injury independent of changes in long chain acylcarnitine. Circ Res. 1988;63(6):1036-1043.

46. Zarain-Herzberg A, et al. Modification of sarcoplasmic reticulum gene expression in pressure overload cardiac hypertrophy by etomoxir. FASEB J. 1996;10(11):1303-1309.

47. Turcani M, Rupp H. Etomoxir improves left ventricular performance of pressure-overloaded rat heart. Circulation. 1997;96(10):3681-3686.

48. Turcani M, Rupp H. Modification of left ventricular hypertrophy by chronic etomixir treatment. Br J Pharmacol. 1999;126(2):501-507.

49. Schmidt-Schweda S, Holubarsch C. First clinical trial with etomoxir in patients with chronic congestive heart failure. Clin Sci (Lond). 2000;99(1):27-35.

50. Holubarsch CJ, et al. A double-blind randomized multicentre clinical trial to evaluate the efficacy and safety of two doses of etomoxir in comparison with placebo in patients with moderate congestive heart failure: the ERGO (etomoxir for the recovery of glucose oxidation) study. Clin Sci (Lond). 2007;113(4):205-212.

51. Plenter RJ, Grazia TJ. Murine heterotopic heart transplant technique. J Vis Exp. 2014;(89):51511.

52. Yao $\mathrm{CH}$, et al. Identifying off-target effects of etomoxir reveals that carnitine palmitoyltransferase I is essential for cancer cell proliferation independent of $\beta$-oxidation. PLoS Biol. 2018;16(3):e2003782. 
53. Yao $\mathrm{CH}$, et al. Dose-response metabolomics to understand biochemical mechanisms and off-target drug effects with the TOXcms software. Anal Chem. 2020;92(2):1856-1864.

54. Divakaruni AS, et al. Etomoxir inhibits macrophage polarization by disrupting CoA homeostasis. Cell Metab. 2018;28(3):490-503.

55. Garcia MR, et al. Monocytic suppressive cells mediate cardiovascular transplantation tolerance in mice. J Clin Invest. 2010;120(7):2486-2496

56. Conde P, et al. DC-SIGN(+) macrophages control the induction of transplantation tolerance. Immunity. 2015;42(6):1143-1158.

57. Braza MS, et al. Inhibiting inflammation with myeloid cell-specific nanobiologics promotes organ transplant acceptance. Immunity. 2018;49(5):819-828.

58. Galluzzi L, et al. Metabolic targets for cancer therapy. Nat Rev Drug Discov. 2013;12(11):829-846.

59. Karwi QG, et al. Loss of metabolic flexibility in the failing heart. Front Cardiovasc Med. 2018;5:68

60. Adamo L, et al. Myocardial B cells are a subset of circulating lymphocytes with delayed transit through the heart. JCI Insight. 2020;5(3):134700

61. Chatur S, et al. Inhibition of vascular endothelial growth factor reduces cardiac allograft vasculopathy. J Heart Lung Transplant 2016;35(9):1124-1130.

62. Gelman AE, et al. Cutting edge: acute lung allograft rejection is independent of secondary lymphoid organs. J Immunol. 2009;182(7):3969-3973.

63. Yu YR, et al. A protocol for the comprehensive flow cytometric analysis of immune cells in normal and inflamed murine non-lymphoid tissues. PLoS One. 2016;11(3):e0150606.

64. McGarry JD, et al. Carnitine palmitoyltransferase I. The site of inhibition of hepatic fatty acid oxidation by malonyl-CoA J Biol Chem. 1978;253(12):4128-4136.

65. McGarry JD, et al. Observations on the affinity for carnitine, and malonyl-CoA sensitivity, of carnitine palmitoyltransferase $\mathrm{I}$ in animal and human tissues. Demonstration of the presence of malonyl-CoA in non-hepatic tissues of the rat. Biochem J. 1983;214(1):21-28.

66. Pande SV, et al. Differential effects of phosphatidylcholine and cardiolipin on carnitine palmitoyltransferase activity. Biochim Biophys Acta. 1986;877(2):223-230. 